\title{
Superior sagittal sinus thrombosis after spinal anesthesia
}

Tareef S. Daqqaq, MD, Abdulelah A. Naqshabandi, $M D, A B P N$, Emad S. Rajih, MD, MPH.

\begin{abstract}

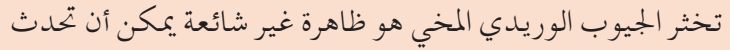

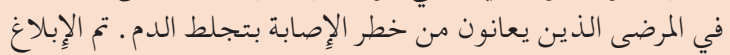

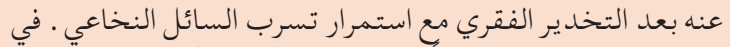

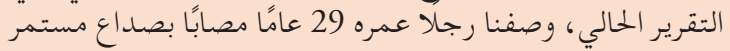

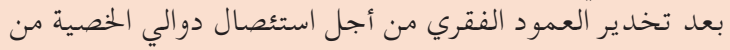

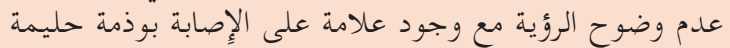

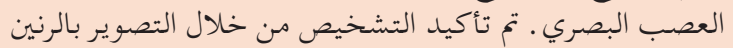

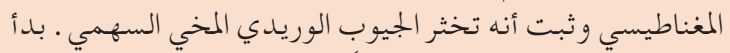

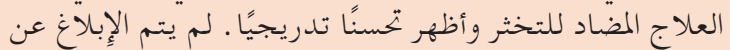

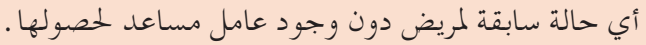

Superior sagittal sinus thrombosis is an uncommon phenomenon that could occur in patients with a risk for thrombosis. It has been reported after spinal anesthesia with persistent cerebrospinal fluid leak. The current case is a young 29-year-old man who was complaining of persistent headache after spinal anesthesia for varicocelectomy and a new onset of blurred vision with a sign of papilledema. The diagnosis was confirmed with magnetic resonance imaging and proved to be superior sagittal sinus thrombosis. He was started on anticoagulant therapy and showed gradual improvement. No previous case has been reported in the literature in a patient without prothrombotic status risk.

Neurosciences 2020; Vol. 25 (3): 210-213 doi: $10.17712 / n s j .2020 .3 .20200003$

From the Department of Urology (Rajih), Department of Radiology (Daqqaq), College of Medicine, Taibah University, Madinah Al-Munawarah, Department of Neurology (Nagshabandi), United Clinics Medical Complex, Madinah Al-Munawarah, Kingdom of Saudi Arabia

Received 8th January 2020. Accepted 26th March 2020.

Address correspondence and reprint request to: Dr. Emad S. Rajih, Department of Urology, College of Medicine, Taibah University, Madinah Al-Munawarah, Kingdom of Saudi Arabia

E-mail:remad@taibahu.edu.sa

ORCID ID: https://orcid.org/0000-0002-4641-8248
Cerebral venous sinus thrombosis (CVT) is an uncommon alarming pathological process. Moreover, women affected more commonly than men and the incidence in annual basis is about 0.9 per $100,000 .^{1,2}$ It commonly occurs secondarily to a genetic or acquired hypercoagulable state such as pregnancy, malignancy, infection, trauma, mechanical heart valve, and oral contraceptive use. ${ }^{3}$ Cerebral venous sinus thrombosis has variable clinical presentations. The most common presenting symptom is headache that can be accompanied with vomiting, papilledema, visual disturbance, and/or focal neurological syndrome such as focal neurological deficit with or without seizures. It can also associate with mental status changes such as stupor or with coma in some occasions. ${ }^{2,3}$

The positional headache considered as the common presenting neurological symptoms after spinal anesthesia, and it occurs in 10 to $30 \%$ of patients. The mechanism behind it, is secondary to persistent cerebrospinal fluid leakage after epidural anesthesia. The course of the headache is usually benign in nature with almost complete recovery a few weeks. A rare complication can occur, which is secondary to brain stem compression such as subdural hygroma and neurological deficits. ${ }^{4}$ It has been reported that CVT rarely occurs in post-partum women after epidural anesthesia who have a tendency for thrombosis secondary to hypercoagulable state. ${ }^{4}$ Herein, we report a rare occurrence of superior sagittal sinus thrombosis in a healthy young man without prothrombotic status who underwent a day surgery procedure with microscopic varicocele ligation, which was performed under spinal anesthesia.

Case Report. Patient information and clinical findings. A young 29-year-old healthy man who came

Disclosure. The authors declare no conflicting interests, support or funding from any drug company. 
to the Neurology Clinics, United Clinics in Madinah Al-Munawarah, at day three with a persistent positional headache after microscopic varicocelectomy for primary infertility. It was associated with nausea and low back pain at the site of spinal puncture. The headache was global type and improved temporarily with hydration and oral caffeine intake. During follow-up, he presented to the Emergency Department with still recurrent headache and new onset of vomiting and blurred vision at day 10 . There was neither history of trauma nor relevant past or family medical history for thromboembolic disorders; he had not been on any medical therapy before. On examination, he was conscious and oriented with a GCS of $15 / 15$. His body temperature was $37.1^{\circ} \mathrm{C}$, with a heart rate of 86 beats/min. His blood pressure was $133 / 85 \mathrm{mmHg}$, and his respiratory rate was 17 breaths/min. Fundoscopic examination showed bilateral papilledema, and no focal neurological deficits were recognized (Table 1).

Diagnostic assessment. Laboratory investigations revealed a sodium level of $131 \mathrm{mEq} / \mathrm{L}$, blood urea and nitrogen levels of $4.5 \mathrm{mg} / \mathrm{dL}$, a creatinine level of 55 micromole/L, and a level of glucose was $10^{9} \mathrm{mg} / \mathrm{dL}$. A complete blood count revealed a normal white blood cell count of $8 \times 10^{9} / \mathrm{L}$, a hemoglobin level of $12.9 \mathrm{~g} / \mathrm{dL}$, and a platelet level of $356 \times 10^{\circ} / \mathrm{L}$. The liver function test was normal, with an alanine aminotransferase (ALT) level of $33 \mathrm{U} / \mathrm{L}$, an aspartate aminotransferase (AST) level of $16 \mathrm{U} / \mathrm{L}$, a bilirubin value of $2 \mathrm{mg} / \mathrm{dL}$, and an alkaline phosphatase (ALP) level of 103 U/L. Further hematological workup revealed normal levels of protein $\mathrm{C}$ and S. His D-dimer level was $2.1 \mathrm{nmol} / \mathrm{L}$, with a fibrinogen level of $3.1 \mathrm{~g} / \mathrm{L}$. His partial thromboplastin time was 35 seconds and high prothrombin time was 11.1 seconds. Anticardiolipin and anti-lupus antibodies were negative. Fasting plasma homocysteine level is within normal range $(13.4 \mu \mathrm{mol} / \mathrm{L})$. Antithrombin III activity level is $114 \%$ which is within normal limit. The sickle cell screening is negative. The total cholesterol is $6.4 \mathrm{mmol} / \mathrm{l}$, triglyceride $1.85 \mathrm{mmol} / \mathrm{l}$, high density lipoprotein is $1.14 \mathrm{mmol} / \mathrm{l}$, and low-density lipoprotein is $5.1 \mathrm{mmol} / \mathrm{l}$. Further genetic tests that include factor $\mathrm{V}$ Leiden and prothrombin gene mutation were negative.

Magnetic resonance (MR) imaging showed increase signal intensity in both T1 weighted images (WI) and T2WI as well as bilateral superficial cortical veins with filling defect as seen in the magnetic resonance venography (MRV); there was no diffusion restriction suggesting venous infarction (Figure 1). Findings are consistent with superior sagittal sinus thrombosis.

Therapeutic intervention. He required admission to the hospital to observe and stabilize him. He was started on heparin infusion and warfarin until the target INR level reached 2-3. On day 8 after admission, the patient showed complete resolution of headache and vomiting. He still had a blurred vision and he was started on acetazolamide $250 \mathrm{mg}$ daily dose for 3 months only. His symptoms gradually improving over time until complete recovery by the end of the second month after discharge.

Follow-up and outcome. His last follow-up was 4 months after surgery and was asymptomatic and his further genetic tests were unremarkable. His INR was maintained between 2 to 3 through all visits to coagulation clinic. He is going to undergo MR imaging follow-up by the end of the 6th month prior to stop anticoagulation.

Table 1 - Timetable demonstrating the sequence of events and case progression

\begin{tabular}{|c|c|c|c|}
\hline Dates & \multicolumn{3}{|c|}{ Relevant past medical history and interventions } \\
\hline 29-9-2019 & \multicolumn{3}{|c|}{$\begin{array}{l}\bullet \text { The patient had history of primary infertiltiy with azospermia. } \\
\bullet \text { He underwent left side microscopic varicocelectomy under spinal anesthesia as day surgical procedure. } \\
\bullet \text { Unremarkable family and social history. }\end{array}$} \\
\hline Dates & Summaries from Initial and Follow-up Visits & $\begin{array}{r}\text { Diagnostic Testin } \\
\text { (including dates) }\end{array}$ & Interventions \\
\hline 2-10-2019 & $\begin{array}{l}\bullet \text { Admitted to the hospital with drowsines and sever } \\
\text { persistent headach for conservative measures. } \\
\text { • He was discharged home in days } 2 \text { in a good condition. }\end{array}$ & $\begin{array}{l}\text { •Complete blood count } \\
\text { • Serum electrolyte }\end{array}$ & $\begin{array}{l}\bullet \text { Intravenous hydration } \\
\bullet \text { Intravenous Paracetamol } \\
\bullet \text { Intravenous metoclopramide } \\
\bullet \text { Non-steroidal anti-inflammatory drug }\end{array}$ \\
\hline $10-10-2019$ & $\begin{array}{l}\text { - Readmitted to the hospital with Sever headach, vomiting, } \\
\text { and papilledema. }\end{array}$ & $\begin{array}{l}\bullet \text { MRI and MRV } \\
\bullet \text { Coagulation study } \\
\bullet \text { Thromboembolic blood work }\end{array}$ & $\begin{array}{l}\bullet \text { Heparin infusion } \\
\bullet \text { Warfarin was started }\end{array}$ \\
\hline 25-12-2019 & $\bullet$ His symptoms was improved and target INR was acheved & $\begin{array}{l}\text { - Repeated Thromboembolic } \\
\text { work-up }\end{array}$ & •Continue on Warfarin \\
\hline
\end{tabular}




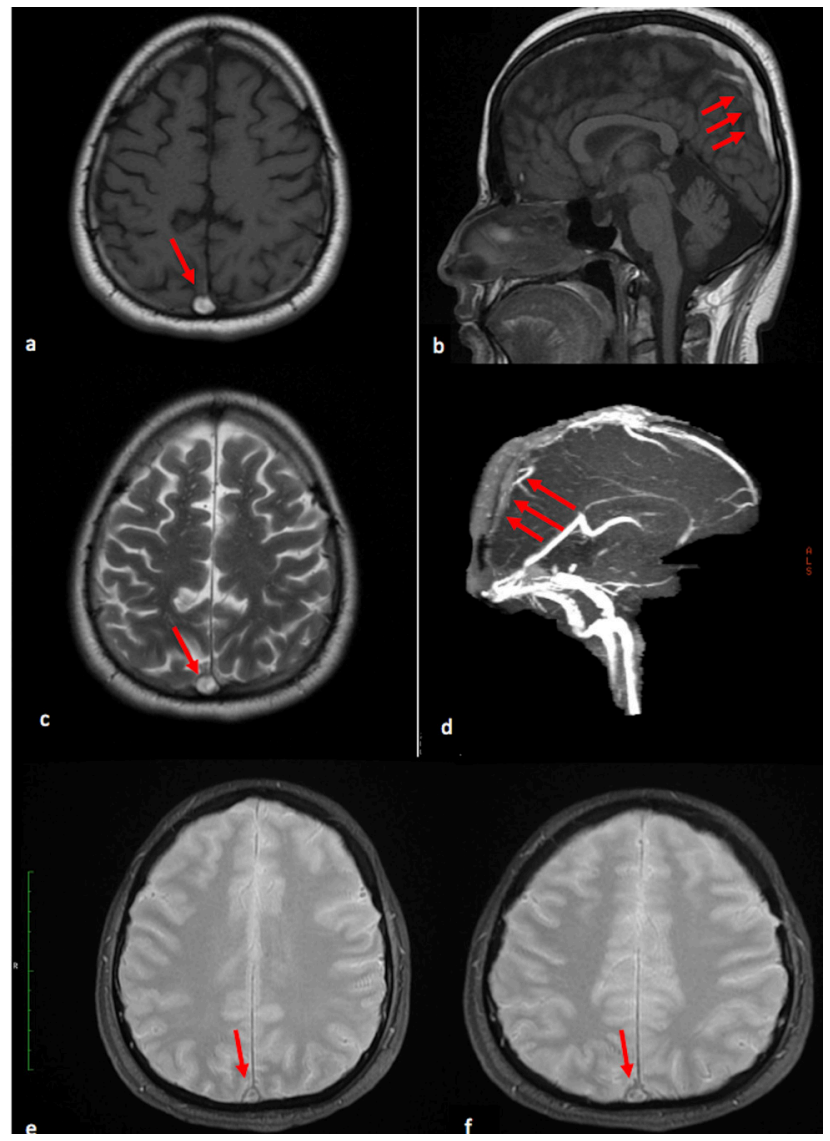

Figure 1 - Subacute thrombus of the superior sagittal sinus. a, b) Axial and sagittal non-contrast T1- weighted, c) axial T2 weighted MR images show an area of abnormal increased signal intensity in the superior sagittal sinus (arrows). d) Sagittal source image from non-contrast- MR venography shows filling defects (arrows) due to a thrombus. e, f) axial T2* GRE weighted MR images shows an area of abnormal low signal intensity (blooming artefact) in the superior sagittal sinus (arrows)

Discussion. The current study reports a case of superior sagittal sinus thrombosis after spinal anesthesia in a patient without prothrombotic risk status, which describes this rare finding in a healthy patient. The current finding was diagnosed by brain MR and MRV imaging studies. Our patient was complaining of severe persistent headache in the first day after surgery. He was readmitted for conservative measures on day 4 after surgery and was started on intravenous fluid and analgesics, with temporary improvement. He was discharged from the hospital in an acceptable condition. Despite these conservative measures, he had progressive severe symptoms after 2 weeks that mandated further imaging studies of the brain, showing superior sagittal sinus thrombosis. He was admitted to the hospital for anticoagulation and further diagnostic thromboembolic workups, albeit with negative results.

Using a large gauge needle and/or multiple punctures during spinal anesthesia will predispose the CSF space to persistent drainage and leak. This condition causes loses of the spinal fluid which can lead to stretching and dilatation of the cerebral vascular systems. Consequently, this will lead to fragility of the venous endothelium secondary to stretching of the cerebral vessels due to negative pressure. Furthermore, reduction in the absorption of the fluid into the sinuses leads to increase the viscosity. ${ }^{2}$ It is also proved in the study done by Canhao P in 2005 that the velocity of the venous blood flow is markedly decreased after spinal anesthesia as it was measured by transcranial doppler ultrasound. ${ }^{5}$ So, many theoretical processes can contribute to the occurrence of the thrombosis that can explain the condition in our patient who has no prothrombotic risk.

Brain MR and MRV imaging was supportive of the diagnosis, showing classical features of superior sagittal sinus thrombosis and cortical venous thrombosis. The mechanism of the CVT in such patients were poorly understood. ${ }^{3,6}$ However, the mechanism behind that thought to be secondary to thrombus in the cerebral veins, that can cause obstruction of the blood drainage from the brain, this in turn can lead to increase venous pressure and affection of the blood-brain barrier. Eventually, this leads to decrease cerebrospinal fluid absorption and elevation of intracranial pressure, which can explain the positional headache and the papilledema. ${ }^{3,6}$

Different case reports addressed the occurrence of sinus thrombosis in anecdotal cases after spinal anesthesia. However, these reported cases occurred among patients with a high risk for thrombosis who underwent spinal anesthesia. Our patient, however, had no identifiable risk for thrombosis. Mahesh ${ }^{7}$ and his associates described a case of A caesarean section was done for a young 31-year-old post-partum woman under spinal anesthesia, in which she developed superior sagittal sinus thrombosis secondary to protein $\mathrm{C}$ and $\mathrm{S}$ deficiency. In their report, they described the pathophysiological aspect of venous thrombosis after intracranial lowpressure system. Furthermore, they studied 52 cases and categorized them into an obstetric-associated group and a non-obstetric-associated group. ${ }^{7}$ This considered in the literature as the largest series describing the natural history of this phenomenon that occurred in presence of at least a single risk factor for sagittal venous sinus thrombosis. Yildiz and his group ${ }^{8}$ reported a series of 
three cases with intracranial hypotension after spinal anesthesia followed by CVT. However, all of them had at least one thromboembolic risk factor. Accordingly, previous studies identified different risk factors for cerebral venous sinus thrombosis after spinal anesthesia. However, our study supports the approach to image the brain and to suspect CVT in case of persistent severe symptoms, even when no identifiable risk factor could be recognized.

The use of magnetic resonance imaging (MRI) sequences (such sequences like T1WI, T2WI, fluid-attenuated inversion recovery (FLAIR), and diffusion-weighted imaging (DWI)) in combination with magnetic resonance venography (MRV) have been largely replaced the use of cerebral angiography and computed tomography (CT), which allows the detection of venous occlusion along with any complications secondary to venous occlusion, such as cerebral edema and areas of venous infarction. ${ }^{1,9}$ The use of MRI allows localization of the thrombus within the venous systems by altering the signal intensity in the MRI sequences secondary to disruption in flow due to thrombus. Thrombus in acute stage can lead to a highly subtle abnormal signal intensity on non-contrast MRI, which can be confused with a normal flow void on T2WI. Although, the isointense signal intensity to brain parenchyma in T1WI and low signal intensity in T2WI indicates that the thrombus in acute stage. When the signal intensity is high in T1WI and is low in T2WI this indicate that the thrombus in early subacute phase, while when the signal is high in both T1WI and T2WI this indicates that the thrombus in late subacute phase as seen in our case (Figure $\mathbf{1} \mathbf{a}, \mathbf{b} \& \mathrm{cc}$ ). The subacute phase takes between 7 to 14 days after that the thrombus become chronic and gives a low to isointense signal in T1WI and high signal in T2WI, which mainly related to vascularization of the connective tissue. ${ }^{1,9}$ Here, MRI and MRV are not only helpful diagnostic tools, but also allow to assess the improvement and recanalization of the venous system in follow-up patients.

Spinal anesthesia is increasingly used in clinical practice, e.g., in pelvic surgical procedures. The current report elaborates the possibility of such complication after spinal anesthesia in the era of advancement of regional anesthesia for different pelvic procedures, especially for day-surgical procedures on young patients with a high risk for headache after spinal anesthesia. This condition could be decreased with the use of a small gauge needle during spinal anesthesia, avoiding multiple punctures to the canal.

In conclusion, our findings indicate the serious effect of severe persistent spinal leak after spinal anesthesia, which could lead to sinus thrombosis even in a patient without risk for prothrombotic state. Persistent severe symptoms after spinal anesthesia should be recognized by the medical team to proceed for brain imaging to enable timely diagnosis and optimize the medical condition with less complications secondary to superior sagittal sinus thrombosis.

Acknowledgement. The authors would like to thank Proof-Reading-Service.com (www.proof-reading-service.com) for English language editing. Further, this research holds no conflict of interest and is not funded through any source.

\section{References}

1. Almqvist H, Mazya M, Delgado AF, Delgado AF. Radiological evaluation in patients with clinical suspicion of cerebral venous sinus thrombosis presenting with nontraumatic headache - a retrospective observational study with a validation cohort. BMC Med Imaging 2020; 20: 24.

2. Guner D, Tiftikcioglu BI, Uludag IF, Oncel D, Zorlu Y. Dural puncture: An overlooked cause of cerebral venous thrombosis. Acta Neurol Belg 2015; 115: 53-57.

3. Wang A, Roten R, Le J. Intracranial Hemorrhage with Cerebral Venous Sinus Thrombosis. J Emerg Med 2019; 56: e59-e60.

4. Dornelles MA, Pereira LM. Dural sinus thrombosis following epidural analgesia for delivery: a clinical case. Brazilian J Anesthesiol 2018; 68: 303-306.

5. Canhão P, Batista $P$, Falcão F. Lumbar puncture and dural sinus thrombosis - A causal or casual association? Cerebrovasc Dis 2005; 19: 53-56.

6. Coutinho JM, Ferro JM, Canhão P, Barinagarrementeria F, Cantú C, Bousser MG, et al. Cerebral venous and sinus thrombosis in women. Stroke 2009; 40: 2356-2361.

7. Kate MP, Thomas B, Sylaja PN. Cerebral venous thrombosis in post-lumbar puncture intracranial hypotension: Case report and review of literature. F1000Research 2014; 3: 1-6.

8. Yildiz OK, Balaban H, Cil G, Oztoprak I, Bolayir E, Topaktas S. Isolated cortical vein thrombosis after epidural anesthesia: Report of three cases. Int J Neurosci 2010; 120: 447-450.

9. Sadigh G, Mullins ME, Saindane AM. Diagnostic performance of MRI sequences for evaluation of dural venous sinus thrombosis. Am J Roentgenol 2016; 206: 1298-1306. 